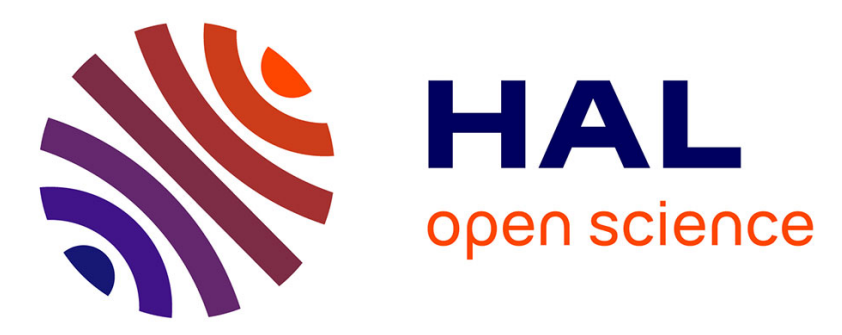

\title{
Large surface micromegas with embedded front-end electronics for a digital hadronic calorimeter
}

\author{
C. Adloff, A. Espargilière, Y. Karyotakis
}

\section{To cite this version:}

C. Adloff, A. Espargilière, Y. Karyotakis. Large surface micromegas with embedded front-end electronics for a digital hadronic calorimeter. 2008 Nuclear Science Symposium, Medical Imaging Conference and 16th Room Temperature Semiconductor Detector Workshop, Oct 2008, Dresden, Germany. pp.1433-1435, 10.1109/NSSMIC.2008.4774684 . in2p3-00363907

\section{HAL Id: in2p3-00363907 https://hal.in2p3.fr/in2p3-00363907}

Submitted on 24 Feb 2009

HAL is a multi-disciplinary open access archive for the deposit and dissemination of scientific research documents, whether they are published or not. The documents may come from teaching and research institutions in France or abroad, or from public or private research centers.
L'archive ouverte pluridisciplinaire HAL, est destinée au dépôt et à la diffusion de documents scientifiques de niveau recherche, publiés ou non, émanant des établissements d'enseignement et de recherche français ou étrangers, des laboratoires publics ou privés. 


\title{
Large surface micromegas with embedded front-end electronics for a digital hadronic calorimeter
}

\author{
C. Adloff, A. Espargilière, Y. Karyotakis \\ LAPP - Université de Savoie - CNRS/IN2P3 \\ BP. 110, F-74941 Annecy-le-Vieux Cedex, France
}

Presented by C. Adloff at

2008 Nuclear Science Symposium, Medical Imaging Conference and

16th Room Temperature Semiconductor Detector Workshop

Dresden (Allemagne), 19-25 october 2008 


\title{
Large Surface MicroMegas with Embedded Front-End Electronics for a Digital Hadronic Calorimeter
}

\author{
Catherine Adloff, Ambroise Espargilière and Yannis Karyotakis
}

\begin{abstract}
In order to study the advantages of a digital hadronic calorimeter for particle flow algorithms, we aim to build a detector prototype with MicroMegas chambers. The bulk technology was chosen for its robustness and the possibility of industrial manufacturing process for mass production. First tests of $1 \mathrm{~cm}^{2}$ granularity MicroMegas with analog readout are very promising. Larger chambers with embedded digital front-end electronics together with detector interface readout boards are being designed. The challenge also lies in the mechanical design of a $1 \mathrm{~m}^{2}$ chamber with a total thickness of $6 \mathrm{~mm}$.
\end{abstract}

\section{INTRODUCTION}

$\mathrm{C}$ URRENT experimental measurements as well as theoretical arguments let predict the existence of new physics at a scale lower than $1 \mathrm{TeV}$. The startup of LHC will allow the exploration of this energy domain, with the aim to discover new phenomena. However, precision measurements will be achieved at a future electron-positron linear collider with energy around $1 \mathrm{TeV}$. This latter is a compulsory continuation to bring the convincing response to the elecroweak symmetry breaking, SUSY models, extra dimensions models, and more generally to any other new physics.

The now common and worldwide project International Linear Collider (ILC) generates a considerable effort on detector R\&D, simulation, and understanding physics signals and backgrounds [1]. The Particle Flow Algorithm (PFA) is the way forward. It requires imaging calorimeters (short radiation-interaction length and high segmentation) located inside the magnetic coil. With about $3000 \mathrm{~m}^{2}$ and 30 Million channels, a good choice for the hadronic calorimeter is a gaseous active medium with embedded digital readout, the socalled digital Hardronic CALorimeter (DHCAL). It has to provide high MIP efficiency, low multiplicity as well as no performance degradation due to high rate, hadronic showers and aging. Technologically, the challenges come from the large area (up to $\left.1.8 \times 3.5 \mathrm{~m}^{2}\right)$ and the little thickness $(<8 \mathrm{~mm})$ of a single gaseous detector, the ease of calibration and the low cost.

The Micro Mesh gaseous structure (MicroMegas) is a gaseous detector, based on the micro-pattern detector technology [2], today widely used by many experiments: COMPASS, CAST, NA48, n-TOF, T2K and ILC TPC project. Our prototypes consist of a commercially available

Manuscript received November 22, 2008. This work was performed within the CALICE collaboration.

C. Adloff, A. Espargilière and Y. Karyotakis are with the University of Savoie and the Laboratoire d'Annecy-le-vieux de Physique des Particules, Annecy-le-Vieux, F-74940 France (e-mail: Catherine.Adloff @lapp.in2p3.fr). fine mesh which separates the drift gap $(3 \mathrm{~mm})$ from the amplification gap $(128 \mu \mathrm{m})$. This simple structure allows full efficiency for MIPs and thanks to thin pillars, provides a good uniformity over the whole surface. The rate obtained with Micromegas chamber is not constrained, as it is the case for the Glass RPC. Moreover the tiny size of the amplification avalanche results in fast signals without physical cross talk and leads to low multiplicities. The chosen bulk technology based on industrial PCB processes, offers a robust detector with working voltages lower than $500 \mathrm{~V}$. MicroMegas are therefore a very appealing possibility to equip a DHCAL optimized for the PFA.

\section{PROTOTYPES DESCRIPTIONS}

Three different kinds of prototypes with $1 \mathrm{~cm}^{2}$ pad size were built at LAPP. One type with analog readout for characterization, two types with embedded digital ASICs.

The analog readout is performed with boards from the CEA laboratory equipped with 6 GASSIPLEX chips (96 channels in total), connected to VME ADCs and featuring high resolution charge determination (12 bits, $0.4 \mathrm{fC}$ per ADC Count). The data acquisition is performed by the CENTAURE program from SUBATECH-Nantes [3]. Three MicroMegas with 6x96 pads and one MicroMegas with 12x32 pads (see Fig. 1) were designed for this analog readout.

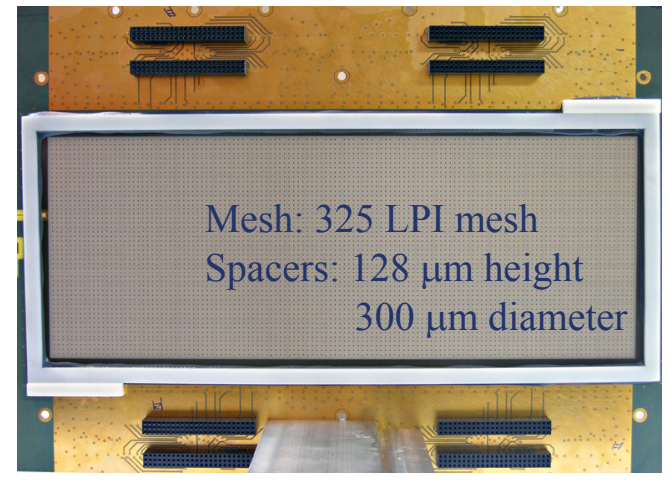

Fig. 1. MicroMegas $12 \times 32 \mathrm{~cm}^{2}$ prototype with 384 pads of $1 \mathrm{~cm}^{2}$ and designed for analog readout.

Two mixed-signal ASICs are foreseen for the digital readout, the HARDROC [4] and the DIRAC [5]. The former was chosen for the baseline of the $1 \mathrm{~m}^{3}$ European DHCAL project in order to get rapidly the digital readout of either MicroMegas or Glass RPC. Whereas the latter is a longer term R\&D which aims to obtain a low cost ASIC with an easy 
signal routing implementation on the detector $\mathrm{PCB}$, an easy calibration and a digital readout down to MicroMegas MIP charges.

All MicroMegas bulks are realized by lamination at high temperature of photosensitive foils and a mesh laid on a PCB with different signal routing depending on the readout. By a photolithographic method, the photo resistive material is etched producing the $128 \mu \mathrm{m}$ pillars. A thin copper foil, glued to part of the calorimeter absorber medium ( $2 \mathrm{~mm}$ thick plate out of a $2 \mathrm{~cm}$ thick absorber), defines the drift cathode. The top of the chamber is therefore not contributing to the active medium thickness. The drift gap is realized with a $3 \mathrm{~mm}$ thick frame which provides also the gas inlets (see Fig. 1).

\section{Radioactive Source Test Results}

Using a ${ }^{55} \mathrm{Fe} X$-ray source, the gain was measured with the analog readout up to 10000 and the energy resolution down to $8.5 \%$ corresponding to a FWHM of $19.6 \%$ (see Fig. 2 and 3). The gain and the FWHM were measured as a function of the drift field, amplification field, gas flow and pressure variables. The expected exponential behavior of the gain versus the amplification field was verified (see Fig. 3) and an absolute pressure dependence of $-2 \mathrm{fC}$ per mbar was determined.

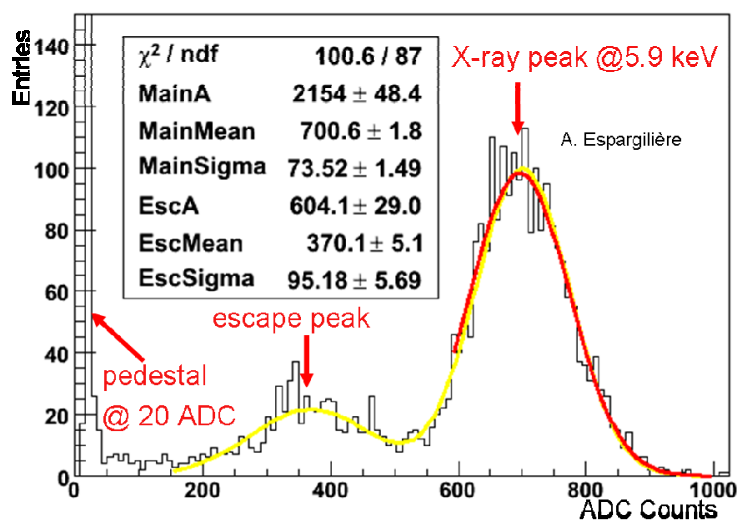

Fig. 2. MicroMegas analog response to a ${ }^{55} \mathrm{Fe} \mathrm{X}$-ray source with $\mathrm{E}_{\text {mesh }}=35$ $\mathrm{kV} / \mathrm{cm}$ and $\mathrm{E}_{\text {drift }}=167 \mathrm{~V} / \mathrm{cm}$. The $5.9 \mathrm{keV}$ emission leads to $700 \mathrm{ADC}$ Counts or $280 \mathrm{fC}$. For a $3 \mathrm{~mm}$ drift gap and a gas mixture of $95 \%$ argon, $5 \%$ isobutane, the gain is about 7600 .

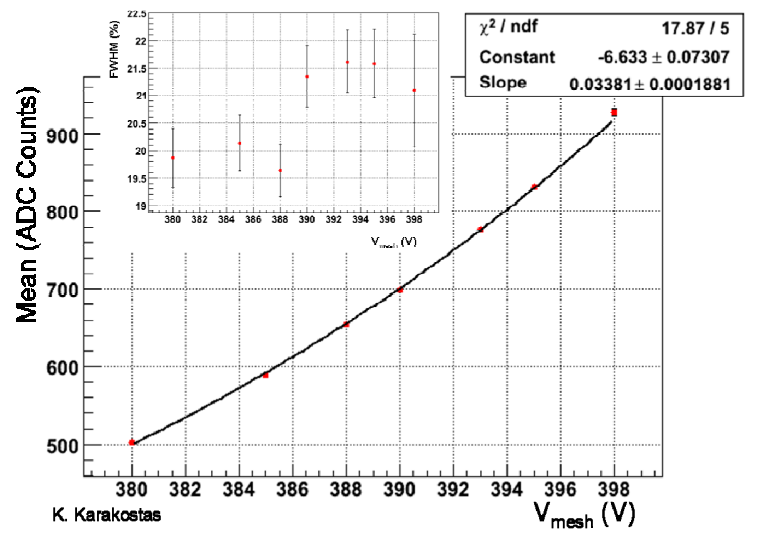

Fig. 3. MicroMegas analog response to a ${ }^{55} \mathrm{Fe} \mathrm{X}$-ray source with $\mathrm{E}_{\text {drift }}=$ $167 \mathrm{~V} / \mathrm{cm}$ : ADC Counts and FWHM versus mesh high voltage.

\section{BEAm TEST RESUlts}

\section{A. Analog Readout Prototypes}

In the summer 2008, four prototypes with analog readout were assembled in a stack and tested at the $\mathrm{H} 2$ line of the SPS-CERN. A total acquisition time of 5 days has allowed to collect data with about 200k muons and 200k pions without absorber plus $250 \mathrm{k}$ pions with an upstream $30 \mathrm{~cm}$ iron block and $1.8 \mathrm{~cm}$ iron absorbers between each prototype.

In the preliminary analysis of the $200 \mathrm{k}$ muons at $200 \mathrm{GeV}$, platinum events were selected by requiring one and only one hit (ADC Counts > 27) in each chamber. The MIP landau distribution on each pad was obtained with a Most Probable Value (MPV) around $45 \mathrm{fC}$. The four chambers mapping was performed in terms of pedestal mean and sigma, MIP landau Most Probable Value (MPV) and sigma. The pedestal gaussian fits have shown very good noise conditions with an average pedestal sigma of $0.6 \mathrm{fC}$. The gain is uniform within each prototype except for the largest one (see Fig. 3). Electronics channel disparity and drift space homogeneity are still to be checked. The next November 2008 test beam has provide the opportunity to upgrade this prototype setup condition.
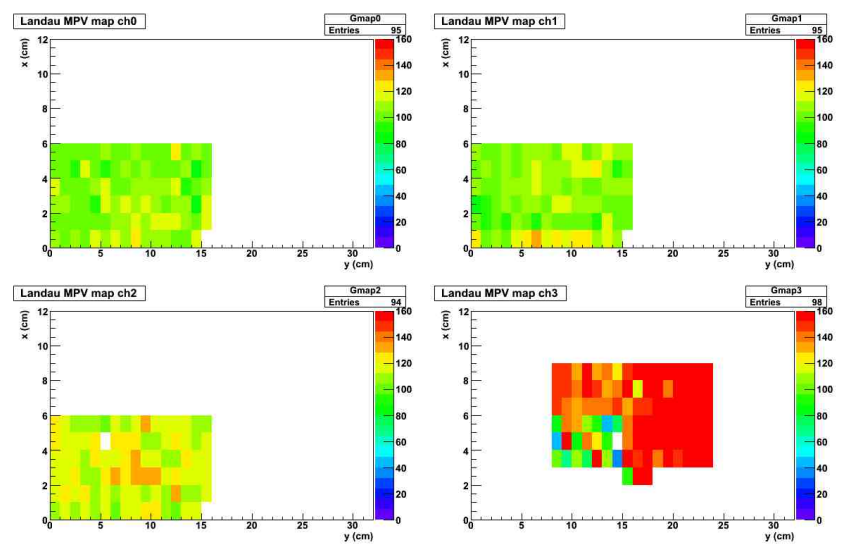

Fig. 3. Chamber mapping of the MicroMegas with analog readout : MPV in ADC counts for golden events selected in a $200 \mathrm{GeV}$ muons sample.

With the same muon sample, golden events were selected by requiring one and only one safe hit (ADC Counts > 51) in three of the four prototypes. Using these three hits, a straight line fit is performed. The efficiency of the last prototype is obtained by looking for at least one hit (ADC Counts $>27$, charge $>2.8 \mathrm{fC}$ ) in a $3 \times 3$ pads square centered around the extrapolated line (see Table I). With the golden events the multiplicity was measured by counting the number of hits in the same square. A multiplicity of about 1.07 was found.

TABLE I

EFFiciency For the MicroMegas Prototypes With ANALOG REAdOUT

\begin{tabular}{|c|c|}
\hline & Efficiency (charge $>2.8 \mathrm{fC})$ \\
\hline Prototype 0 (96 pads) & $97,05 \pm 0,07 \%$ \\
\hline Prototype 1 (96 pads) & $98,54 \pm 0,05 \%$ \\
\hline Prototype 2 (96 pads) & $92,99 \pm 0,10 \%$ \\
\hline Prototype 3 (384 pads) & $96,17 \pm 0,07 \%$ \\
\hline
\end{tabular}




\section{B. First Digital Readout Prototype}

The DIRAC chip was embedded on an $8 \times 8 \mathrm{~cm}$ PCB with additional sparks protection (see Fig 4) [5]. For the first time a prototype with a bulk laid on a PCB with embedded electronics reaching a total thickness of $12 \mathrm{~mm}$ including 2 $\mathrm{mm}$ absorber was operational and exposed to $200 \mathrm{GeV}$ pions at the $\mathrm{H} 2$ beam line in summer 2008. Fig. 5 shows the beam profile with a threshold above $19 \mathrm{fC}$. Further tests with a stack of prototypes are compulsory to measure threshold dependence, efficiencies and multiplicities.
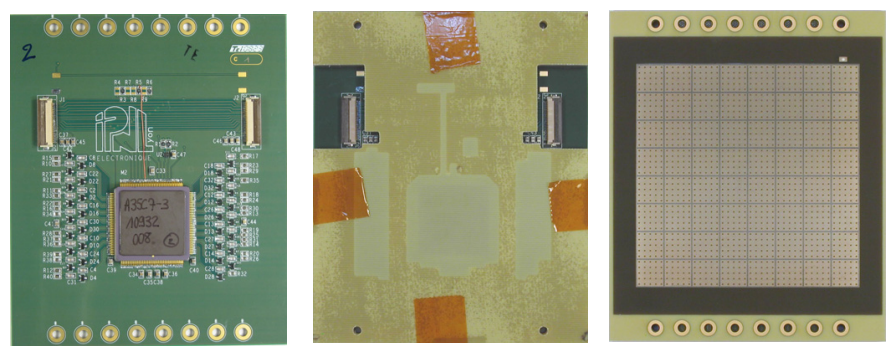

Fig. 4. MicroMegas prototype with DIRAC digital readout. From lest to right: ASIC side, ASIC side with mask for bulk laying and pad side with bulk.

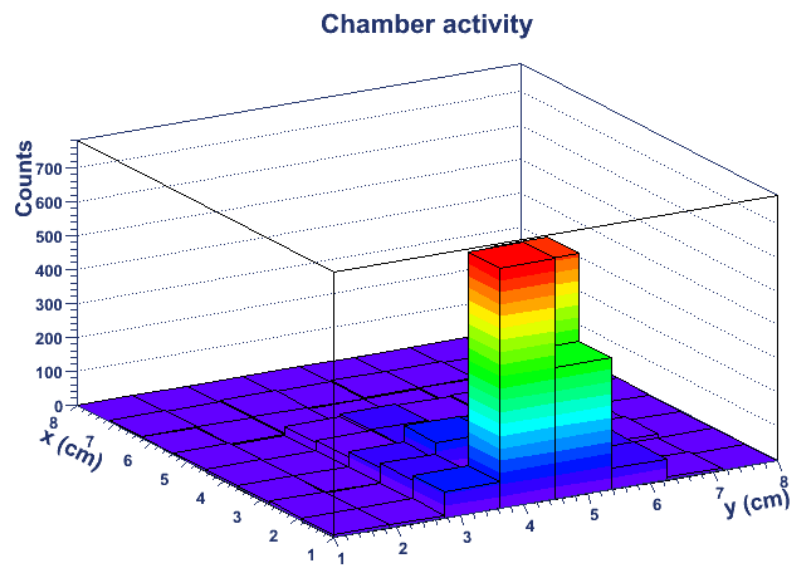

Fig. 5. Chamber mapping of the MicroMegas prototype with DIRAC digital readout : digital Counts in a $200 \mathrm{GeV}$ pion sample.

\section{FUtURE DEVELOPMENTS}

Several new $8 \times 32$ pads prototypes have been realized with four HARDROC chips embedded on one PCB. The electronics is tested with the Detector InterFace board (DIF) which has been designed at LAPP in the frame of the DHCAL CALICE data acquisition system [6]. These prototypes have been exposed to $7 \mathrm{GeV}$ pions during the November 2008 beam test at the T9 line of the PS-CERN, the analysis is ongoing.

The $1 \mathrm{~m}^{2}$ prototype is an assembly of six bulks with 24 ASICs each on a single mask. The chamber is closed by two plates of $2 \mathrm{~mm}$ thick stainless steel (see Fig. 6). This prototype should not exceed a total thickness of $6 \mathrm{~mm}$ (without absorber). It is scheduled to build this prototype beginning 2009 in order to test it in the beam with the former smaller prototypes during late summer 2009 . The $1 \mathrm{~m}^{2}$ design is foreseen for large quantities production in order to build a $1 \mathrm{~m}^{3}$ DHCAL prototype.

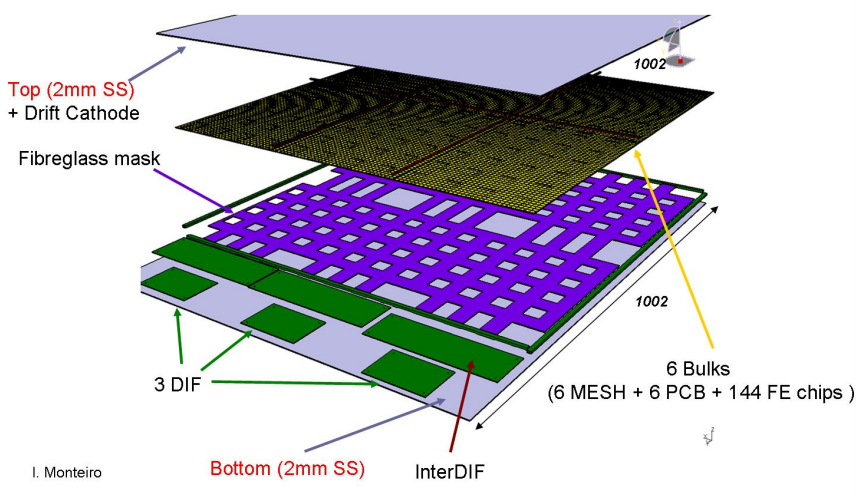

Fig. 6. Design of the $1 \mathrm{~m}^{2}$ MicroMegas Prototype.

\section{SUMMERY AND OUTLOOK}

Several MicroMegas prototypes with analog readout have been successfully built and extensively tested. The summer 2008 MicroMegas beam test results have shown very good performance complying with the DHCAL requirements. First operational bulk MicroMegas with embedded digital electronics was realized and exposed to pion beam. Further studies with a stack of these new extra-thin prototypes are foreseen. Development of a large scale prototype compatible with the CALICE DAQ is well underway to be ready for a beam test during the year 2009 .

\section{ACKNOWLEDGMENT}

We wish to thank Rui de Olivera and the CERN TS-DEM group for the bulk productions and all the advices provided to reach excellent performance prototypes. We also wish to thank Didier Roy from SUBATECH-Nantes for the precious help and work on his DAQ program CENTAURE which allowed us to take the great amount of data during the summer 2008 beam test. We are grateful to Paul Colas, David Attié and Stephen Turnbull from the CEA Laboratory for their fruitful participation to the summer 2008 beam test.

\section{REFERENCES}

[1] ILC Global Design Effort and World Wide Study, International Linear Collider Reference Design Report

[2] Y, Giomataris, Ph. Rebourgeard, J.P Robert and G. Charpak, "MICROMEGAS: A High granularity position sensitive gaseous detector for high particle flux environments", NIM A376, 1996, pp 2935

[3] D. Roy, "CENTAURE Acquisition Program", http://wwwsubatech.in2p3.fr/ electro/infoaq/CENTAURE/main_centaure.html

[4] S. Callier, F. Dulucq, Ch. de La Taille, G. Martin-Chassard, N. SeguinMoreau, R. Gaglione, I. Laktineh, H. Mathez, V. Boudry, J-C. Brient, C. Jauffret, "HARDROC1, readout chip of the Digital HAdronic CALorimeter of ILC", IEEE-NSS Conference Record, vol 3, 2007, pp $1851-1856$

[5] R. Gaglione, "DIRAC: Digital Readout ASIC for hAdronic Calorimeter", IEEE-NSS/MIC 2008

[6] B. Hommels, "Data Acquisition Systems for Future Calorimetry at the International Linear Collider", IEEE-NSS/MIC 2008 